\title{
Coherent Pion Production by Neutrinos
}

\author{
E.A. Paschos \\ University of Dortmund, Institute of Physics, D-44221 Dortmund, Germany
}

\begin{abstract}
I concentrate in this article on the reaction coherent pion production by neutrinos incident on nuclei. A special effort is made in order to describe the approximations entering the calculation. I conclude that the reaction is well understood and with appropriate data for hadronic reactions it can be computed for low and high neutrino energies. Because of shortage of space I omitted the resonance analysis, which is described in articles with my collaborators.
\end{abstract}

Keywords: Coherent Scattering, Neutrino Interactions

PACS: $13.15 .+\mathrm{g}, 14.60 . \mathrm{Pq}$

During the past few years coherent pion production by neutrinos attracted a good deal of attention. There are new experimental results [1, 2] which motivate the interest. Furthermore, there is better theoretical understanding of the process. The theoretical articles use two distinct methods for their analysis. The first method considers the production of the pions in the low $Q^{2}$ and large $v$ region which justifies the application of the PCAC relation [3, 4, 5, 6]. The method is also valid at high neutrino energies, provided the above restriction $\left(v \gg \sqrt{Q^{2}}\right)$ is satisfied. The second method [7, 8] studies the coherent production of the $\Delta$-resonance on nuclei by using a modified $\Delta$-propagator and a distorted wave-function for the pion. My article will concentrate on the first method. I will state the various conditions entering the calculation and then remark on the results of recent articles.

The first method is based on the following steps.

(1) In neutrino-nucleon or -nucleus scattering there is a kinematic region where the dominant term of the leptonic current is proportional to the momentum transfer at the leptonic vertex, denoted by $q_{\mu}[9]$. This region contains the domain

$$
Q^{2}=(\mathrm{a} \mathrm{few}) \cdot \mathrm{m}_{\pi}^{2}
$$

where the PCAC approximation is valid. As a result the vector contribution vanishes (by CVC) and the axial contribution is replaced by a pion-nucleus cross section.

(2) Coherent pion production is defined as the process where the four momentumtransfer squared $(t)$ between the current and the produced pion is small so that the nucleus remains intact. This was the signature of the early bubble chamber experiments - a sharp peak in the $t$-dependence.

As a consequence of step (1) and the above definition, coherent pion production by neutrinos is related to the diffractive peak observed in elastic pion-nucleus scattering. 
In addition to the dominant term of the leptonic current there are subdominant contributions, which in the above kinematic region can be estimated by using data of the reactions $\gamma+N \rightarrow \pi^{0}+N$ and $\pi+N \rightarrow A+N$. This was done recently in [5] where it was established that their contribution is small; justifying a posteriori the above approximations.

I will give several steps of the calculation in order to appreciate the accuracy of the result. We consider the reaction

$$
v\left(k_{1}\right) N(p) \rightarrow \mu^{-}\left(k_{2}\right) \pi\left(p_{\pi}\right) N\left(p^{\prime}\right)
$$

where the momenta are indicated in the parentheses. The invariant amplitude for the process is

$$
T_{W}=\frac{G}{\sqrt{2}} V_{u d} \bar{u}\left(k_{2}\right) \gamma^{\mu}\left(1-\gamma_{5}\right) u\left(k_{1}\right)\left[V_{\mu}^{+}-A_{\mu}^{+}\right] .
$$

For coherent scattering there is no vector $\otimes$ axial inference. The matrix element of the axial current is indicated by $A_{\mu}^{+}$and consists of the pion pole contribution and the rest we call $R_{\mu}$. Therefore

$$
-i A_{\mu}^{+}=\frac{\sqrt{2} f_{\pi} q_{\mu}}{Q^{2}+m_{\pi}^{2}} T\left(\pi+N \rightarrow \pi^{+} N\right)-R_{\mu} .
$$

The PCAC relation now reads [5]

$$
q^{\mu} R_{\mu}=-\sqrt{2} f_{\pi} T\left(\pi^{+} N \rightarrow \pi^{+} N\right) .
$$

In the domain of coherence the variables attain a simple form. In the limit $v \gg \sqrt{Q^{2}}$ we use the approximations

$$
\begin{gathered}
q_{\mu}=\left(q_{0}, 0,0, \sqrt{v^{2}+Q^{2}}\right) \approx\left(v, 0,0, v+\frac{1}{2} \frac{Q^{2}}{v}\right) \\
-q^{2}=Q^{2}=m_{\ell}^{2} \frac{y}{1-y}+k_{1}^{0} k_{2}^{0} \theta^{2} \quad \text { with } \quad y=\frac{v}{E_{1}} .
\end{gathered}
$$

Of particular interest is the experimental configuration $\theta=0$ when the muon is parallel to the neutrino (in the laboratory frame). In this case [9]

$$
Q^{2}=m_{\ell}^{2} \frac{y}{1-y}, k_{1 \mu}=\frac{1}{y} q_{\mu} \quad \text { and } \quad k_{2 \mu}=\frac{1-y}{y} q_{\mu} .
$$

There are also smaller terms in these formulas which have been estimated to give smaller cross sections [5].

The cross section is calculated in a straight-forward way. After squaring the matrix element $T_{W}$ we can explicitly calculate the leptonic tensor in terms of traces. In the parallel configuration we make the substitutions in equation (8), which indicate that 
all surviving lepton terms are proportional to $q_{\mu}$, as stated in item (1) at the beginning of this article. Thus the vector contribution $V_{\mu}^{+}$vanishes, the pion pole is calculated explicitly and $q^{\mu} R_{\mu}$ is determined by PCAC. This way I reproduced the original Adler formula [9]. Comparisons with experimental data require an extrapolation of the formulas to finite values of $Q^{2}$, which is mentioned in the original article [9]. Later on an improved extrapolation was introduced by using for $Q^{2}$ eq. (7) instead of (8) in the square of the pion-pole term [10]. With these steps the original formulas are reproduced and the approximations are evident.

In our work [5] we analysed coherent $t$-pion production in terms of helicity cross sections. We treat the kinematics exactly by calculating the density matrix elements. Among the four polarizations we only approximate the helicity zero polarization as follows

$$
\epsilon_{\mu}(\lambda=0)=\frac{1}{\sqrt{Q^{2}}}\left(|\vec{q}|, 0,0, q_{0}\right) \approx \frac{q_{\mu}}{\sqrt{Q^{2}}}+0\left(\frac{Q^{2}}{v^{2}}\right)
$$

in order to apply the PCAC relation. The other polarizations with exact density matrix elements are kept in the calculation. This is evident in equations (7), (13) and (16) in ref. [5]. For $E_{v}<4 \mathrm{GeV}$ we use experimental data for either calculating or estimating the various terms. We found that the helicity zero and the longitudinal cross sections dominate. Our formulas look more complicated but they are more accurate because they include the explicit muon mass, $Q^{2}$ and $v$ dependence of the density matrix elements.

When we write out the pion pole explicitly, we expect the remaining hadronic cross sections to be smooth functions of $Q^{2}$ for $Q^{2} \lesssim 0.1 \mathrm{GeV}^{2}$. Thus the $Q^{2}$-extrapolation is given by the kinematic factors appearing in our formulas. The presence of the muon mass reduces the charged current cross section in the small $Q^{2}$ domain. This is evident in the functional form of $\tilde{L}_{\mathrm{oo}}$ and $\tilde{L}_{\ell_{\mathrm{o}}}$ in equation (6) of ref. [5]. The net effect is a reduction of the differential cross section $d \sigma / d Q^{2}$ in the small $Q^{2}$ (see figure 2 in [5]). A reduction is also reported in a recent article [6] where the authors use the improved $Q^{2}$ extrapolation [10].

Using our formulas we calculated the differential $d \sigma / d Q^{2}$ and the integrated cross sections. We found a substantial reduction for the $C C$ reaction. The reduction brings better agreement with the K2K upper limit.

To sum up, coherent pion production by neutrinos represents a very attractive reaction which is theoretically well understood. Looking at the derivation I expect that a careful calculation should give a theoretical cross section with an accuracy of 20 to 30\%, which is typical of PCAC predictions. For this reason we are now repeating the low energy $\left(E_{v}<4 \mathrm{GeV}\right)$ calculation and plan to extend it to higher neutrino energies where data is already available. A successful explanation of all data will indicate that we possess an accurate theoretical calculation. Consequently, this reaction together with quasielastic scattering and resonance production will serve in the future as a bench-mark for determining the experimental flux and properties of the neutrinos. 


\section{ACKNOWLEDGMENTS}

I wish to thank Dr. C. Hill and the theory group for their hospitality at Fermilab. I also thank Dr. J. Morfin for organizing a wonderful meeting.

\section{REFERENCES}

1. M. Hasegawa et al. (K2K), Phys. Rev. Lett. 95, 252301 (2005); see also L. Whitehead's contribution to this meeting.

2. J.L. Raaf, Ph.D. thesis, Miniboone [Femilab-report No. 2005.20]; B.T. Flemming's contribution to this meeting.

3. D. Rein and L. Sehgal, Nucl. Phys. B223, 29 (1983).

4. A.A. Belkov and B.Z. Kopeliovich, Sov. J. Nucl. Phys. 46, 499 (1987)

5. G. Gounaris, A. Kartavtsev, and E.A. Paschos Phys. Rev. D74, 054007 (2006).

6. D. Rein and L. Sehgal, arXiv: hep-ph/0606185(2006) and L. Sehgal's contribution to this meeting.

7. S.K. Singh, M.S. Athar, and S. Ahmad, Phys. Rev. Lett. 96, 241801 (2006)

8. L. Alvarez-Ruso, L.S. Geng, and M.J. Vicente Vacas, arXiv:0707.2172 (2007)

9. S.L. Adler, Phys. Rev. 135, B963 (1964).

10. S.L. Adler, arXiv: hep-ph/0505177, pp. 11-12. 